\title{
Complex formation in systems of oppositely charged polyelectrolytes: A molecular dynamics simulation study
}

\author{
Roland G. Winkler \\ Institut für Festkörperforschung, Forschungszentrum Jülich, 52425 Jülich, Germany \\ Martin O. Steinhauser and Peter Reineker \\ Abteilung Theoretische Physik, Universität Ulm, 89069 Ulm, Germany \\ (Received 16 October 2001; published 16 August 2002)
}

\begin{abstract}
Results of molecular dynamics simulations for systems with two flexible, oppositely charged polymer chains are presented. The lengths $N$ and interaction strength $\lambda$ of the chains are varied. We find that the chains remain separated for small values of $\lambda$. For large interaction strengths, i.e., large Bjerrum lengths, we find glasslike structures and order on the length scale of a few monomer diameters. Between these two limits of the interaction strengths, the chains of various lengths collapse into compact complexes that exhibit self-similar structures. The scaling behavior of the radius of gyration is discussed as a function of chain length and interaction strength. In addition, the local structure of the collapsed systems is analyzed and the dependence of the density of the aggregate on the interaction strength is discussed.
\end{abstract}

DOI: 10.1103/PhysRevE.66.021802

PACS number(s): 61.90. $+\mathrm{d}, 36.20 .-\mathrm{r}$

\section{INTRODUCTION}

Polyelectrolytes play a major role in everyday life, particularly in biological systems. However, despite their importance, polyelectrolytes are not as well understood as uncharged polymer systems. At present there is no satisfactory theory for these charged systems at very low salt concentrations. The long-range Coulomb interaction introduces a new length scale in the system and renders analytical calculations difficult [1]. On the experimental side there are various investigations of polyelectrolytes underway, but they are difficult to perform [2-4]. Computer simulations are therefore an important tool for the detailed investigation of these systems on a molecular level. So far, mostly chains with added counterions and colloidal systems have been studied [5-9], but no systematic analysis of systems consisting only of chains have been performed yet. In solutions of oppositely charged polyions, the strong Coulomb attraction leads to the formation of complexes. Such complexes are relevant for a variety of technical applications and are fundamental in biological systems. Of particular interest is the application of complexes of DNA and oppositely charged macromolecules in gene therapy. The problem is to introduce genetic material into cell nuclei. Experiments on DNA complexes indicate conformational changes of the DNA molecule during the complex formation. An example is provided in Ref. [10], where DNA in solution is compacted in the presence of spermidine. Stable complexes are also formed between DNA and cationic lipids (lipoplexes) [11]. Srivastava and Muthukumar [12] studied the structure of aggregates formed by oppositely charged polyelectrolytes, but only for a very small range of interaction strengths and chain lengths.

Polyampholytes, which exhibit to some extent qualitatively similar structures (depending on their charge distribution), have been investigated in more detail [13-24,26]. The structures of such molecules are sensitive to small variations of the positive and negative charges per chain $[15,16]$. Hence, a rich variety of equilibrium conformations is ob- tained reaching from globular states [14] to extended chains $[15,16]$ and pearl-necklace-like sequences $[19,25,26]$. Computer simulations of many-chain polyampholyte systems yield condensed states with glasslike or crystallinelike states depending on the variability of the bonds [24]. Similarly, simulations of diblock polyampholytes (consisting of one positively and one negatively charged block) yield a pairing of the two blocks at large Coulomb interaction strengths and the formation of a coiled state [23]. The effect of an applied electric field on the deformation properties of a polyampholyte was recently studied analytically $[27,28]$ and by simulations [29].

The aim of the simulations presented in this paper is to investigate what kinds of structures are formed by oppositely charged chain systems when they collapse, thereby forming compact clusterlike structures that are physically constrainted to a small region in space. The simulations reach beyond previous ones, since we study systematically a very large range of interaction strengths and chain lengths. In particular, we analyze the scaling behavior of the chain conformations with respect to the chain length. Such investigations are also of fundamental importance for experiments searching ways to produce chemically well-defined nanostructures consisting of polyelectrolytes [30,31]. A deeper theoretical insight into possible structures formed by flexible charged chains might help to improve these experimental endeavors. Our studies focus on two-chain systems. Hence, the results apply to the formation of chain pairs in many-chain systems only. Such pairs certainly form in dilute systems, where the density is much smaller than the overlap concentration. In this limit, the behavior of the solution is determined by the aggregates of two or only a few chains. Depending on the concentration of positively and negatively charged chains, all or only part of the molecules form pairs [32]. Larger aggregates are formed at polymer concentrations close to the overlap concentration. The behavior of the solution is then determined by large globules.

The paper is organized as follows. In Sec. II we describe 
the system and the simulation method. In Sec. III the results are presented. In particular, the dependence of the radius of gyration on the interaction strength between the chains is discussed. In addition, results for the pair correlation function and the density within a cluster are presented. In Sec. IV the kinetics of the cluster formation is outlined. Finally, Sec. $\mathrm{V}$ summarizes our findings.

\section{SYSTEM AND SIMULATION METHOD}

We performed constant temperature molecular dynamics simulations with two oppositely charged chain molecules. The mass points of a chain are connected by harmonic bonds with the potential

$$
V_{B}=\frac{\kappa}{2} \sum_{i}\left(\left|\mathbf{r}_{i}-\mathbf{r}_{i-1}\right|-l\right)^{2},
$$

where $i=1, \ldots, N$. To ensure small fluctuations in the bond lengths (below 1\%), a large value for the force constant $\kappa$ is chosen $\left[\kappa=5000 \epsilon / \sigma^{2}\right.$, where $\epsilon$ and $\sigma$ are the parameters of the Lennard-Jones potential, cf. Eq. (2)].

The finite size of a monomer is taken into account by the truncated Lennard-Jones (LJ) potential

$$
V_{L J}=\left\{\begin{array}{l}
4 \epsilon\left[\left(\frac{\sigma}{r}\right)^{12}-\left(\frac{\sigma}{r}\right)^{6}\right]+\epsilon \quad \text { for } \quad r<\sqrt[6]{2} \sigma, \\
0 \text { for } r>\sqrt[6]{2} \sigma
\end{array}\right.
$$

where $r$ denotes the distance between two (nonbonded) mass points. In our simulation we set the bond length $l=\sigma$.

In addition, the mass points interact via the Coulomb potential

$$
V_{C}=\sum_{i} \sum_{j>i} \frac{z_{i} z_{j} e^{2}}{\varepsilon\left|\mathbf{r}_{i}-\mathbf{r}_{j}\right|}
$$

$\varepsilon$ is the electric permittivity and $e$ the electronic charge. The $z_{i}= \pm 1$ denote the charge state of the monomers. The interaction with the solvent is taken into account by a stochastic force $\left(\boldsymbol{\Gamma}_{i}\right)$ and a friction force, with a damping constant $\gamma$, acting on each mass point. The equations of motion of the system are then given by the Langevin equations

$$
m \ddot{\mathbf{r}_{i}}=\mathbf{F}_{i}-\gamma m \dot{\mathbf{r}}_{i}+\boldsymbol{\Gamma}_{i} .
$$

$\mathbf{F}_{i}$ comprises the force due to the potentials (1)-(3), and $m$ is the mass of a point. The stochastic force is assumed to be stationary, random, and Gaussian (white noise). This insures the proper equilibration of the dilute system $[33,34]$.

The electrically neutral system is placed in a cubic simulation box and periodic boundary conditions are applied for the intermolecular Lennard-Jones interaction, thereby keeping the density $\left(\rho=N / V=2.2 \times 10^{-7} / \sigma^{3}\right)$ of the system constant when changing the chain length. This is only relevant for very low interaction strengths, where the chains are well separated. The number $(N)$ of monomers per chain was chosen as $N=10,20,40,80$, and 160 to span at least one order of magnitude. Hence, it is possible to study the scaling be-

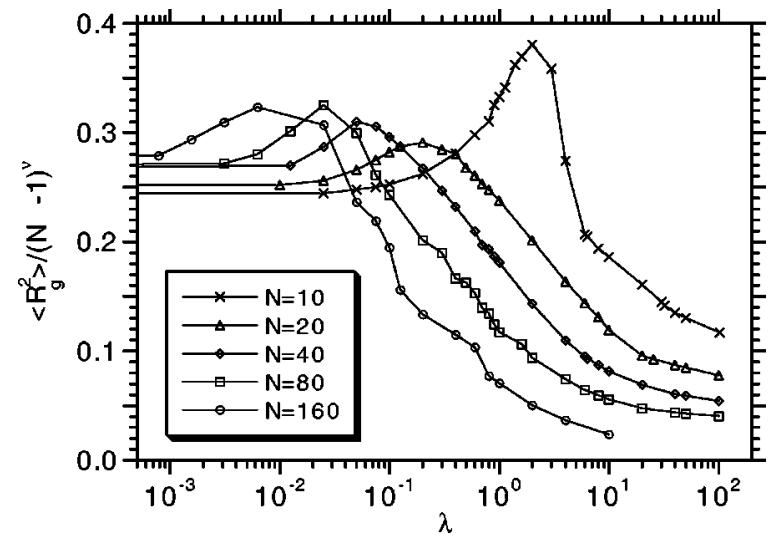

FIG. 1. Radius of gyration as a function of the interaction strength $\lambda$ for various chain lengths $N$. The exponent $\nu$ is $\nu=0.6$.

havior of the systems with respect to size at a given interaction strength for this length scale. In terms of the LennardJones potential parameters $(\sigma, \epsilon)$ the (dimensionless) Coulomb interaction parameter $\lambda$ is given by $\lambda$ $=\lambda_{B} k_{B} T / \epsilon \sigma$, where $\lambda_{B}=e^{2} / \varepsilon k_{B} T$ is the Bjerrum length. Hence, there are only two relevant parameters in our simulation, the length of the chains $N$ and the interaction strength $\lambda$. $\lambda$ is chosen in the range of $\lambda=0, \ldots, 100$. This range covers uncharged chains in good solvent as well as highly charged chain systems. For the Coulomb interaction we use a cutoff $R_{c}$ that is half the box length. This is possible because the eventually analyzed collapsed systems are confined to a small region in space, which is much smaller than $R_{c}$. For noncollapsed chains, the Coulomb interaction is sufficiently weak to be neglected beyond the cutoff distance. The temperature is chosen as $k_{B} T=1.2 \epsilon$, the damping constant as $\gamma=1 / \tau$, and the time step is $\Delta t=5 \times 10^{-3} \tau$, where $\tau$ is the time unit of the simulation. To obtain reasonable statistics, at least 20 different initial conditions were considered and simulations up to $10^{7}$ time steps were performed for each value of $\lambda$ and $N$. An estimate of the errors based upon the standard deviations of these ensembles yields relative uncertainties below $5 \%$.

\section{RESULTS AND DISCUSSION}

\section{A. Radius of gyration}

As a consequence of the long-range nature of the Coulomb interaction, there is no unique length scale in a polyelectrolyte system. As is well known, the radius of gyration $R_{g}^{2}$ and the end-to-end distance $R^{2}$ of neutral chains in good solvent exhibit the universal scaling relation $R^{2} \sim R_{g}^{2} \sim N^{2 \nu}$, with $\nu \approx 0.6$. In case of polyelectrolyte systems, however, the various length scales have to be analyzed individually and we do not expect the same exponents for the radius of gyration and the end-to-end distance for a given interaction strength. A scaling analysis of the conformation of a polyelectrolyte chain in the presence of counterions exhibits indeed length scale dependent scaling behavior [8]. Figure 1 illustrates the radius of gyration of our investigated chains for the whole range of the parameter $\lambda$. Starting from neutral 

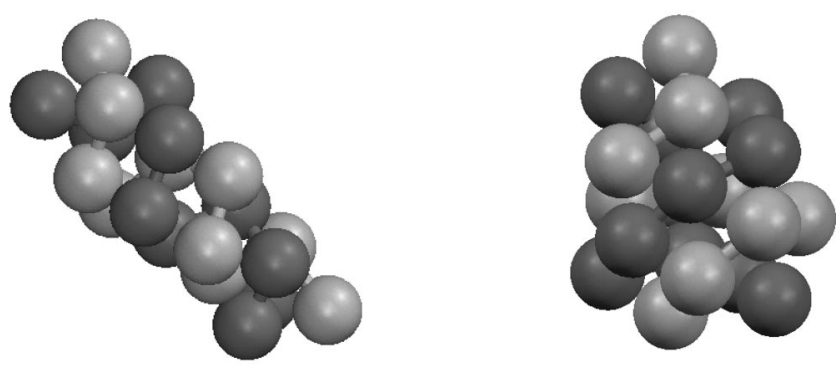

FIG. 2. Conformations of the chains of length $N=10$ for the interaction strength $\lambda=20$.

systems, it is obvious that an increase of $\lambda$ causes the chains to stretch. Beyond a certain value of $\lambda$, the chains start to collapse and $R_{g}^{2}$ decreases. The onset of the collapse obviously depends upon the lengths of the chains. For large interaction strengths, the size of the collapsed chains is smaller than the size of an uncharged chain in a good solvent. There is evidently an attractive interaction in the system present, which gives rise to the compact structure.

The smallest chain systems with $N=10$ do not fit into the scheme of the larger ones. For $N=10$ the ratio $R_{g}^{2} /(N-1)^{\nu}$ exceeds obviously the value for chains of length $N=20$. A more detailed analysis exhibits two conformations for such chains $(\lambda \approx 1)$ : A rather collapsed state (globule) and a stretched state (double-stranded helix). The two conformations are energetically very similar. Therefore, we cannot decide which is the equilibrium state. The appearance of one or the other structure seems to depend strongly on the relative location of the individual monomers. A certain mismatch in the relative positions leads to a collapsed state, whereas in the opposite situation the extended state is stabilized. For large interaction strengths, however, the globular state is assumed. Figure 2 presents snapshots of such systems. Both aggregates are obtained for $\lambda=20$. We will now discuss the scaling behavior in more detail.

\section{Small values of $\lambda$}

To investigate the universal features of the chains of various lengths, it is necessary to compare systems of similar interaction strengths. Depending on the separation of the two chains and their monomers, respectively, from each other, we expect at least three different regimes. The effective Coulomb interaction between far apart chains (very low interaction strength) is proportional to $N^{2}$, because the coils behave like point charges with $N$ charges per coil. With increasing Coulomb interaction strength the coils approach each other and the mean distance is of the order of the radius of gyration. Here we expect a scaling behavior of the form $N^{\phi}$ with $\phi=2-\nu \approx 1.4$ [23]. This simply follows from the fact that the Coulomb energy obeys the relation $E_{C} \sim N^{2} / R_{g} \sim N^{2} / N^{\nu}$ for that regime. The Coulomb energy itself is of the order of $k_{B} T$. Thus, the strength of the interaction is estimated to be $\lambda_{B} N^{\phi} \approx 1$ in the weakly attractive regime. Finally, for very large interaction strength, we will find compact structures. Their scaling behavior will be discussed below.

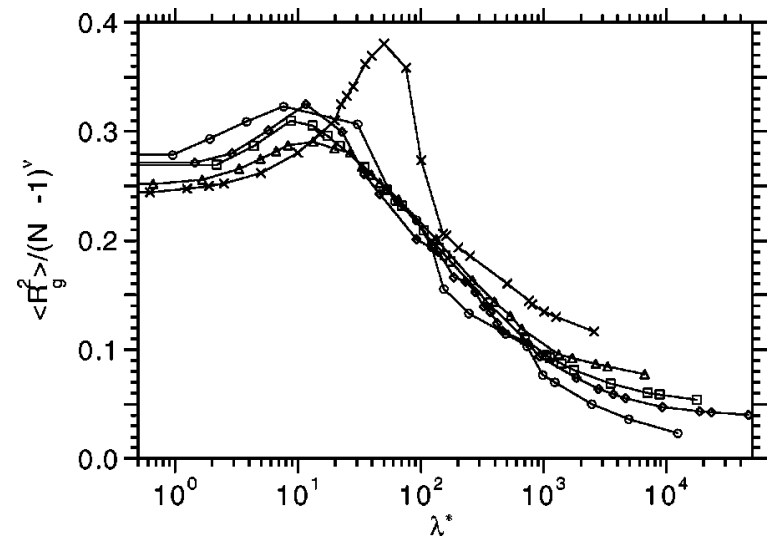

FIG. 3. Radius of gyration as a function of the effective interaction strength $\lambda *=\lambda N^{1.4}$ for various chain lengths $N$ (see Fig. 1). The curves for the various chains lengths $N \geqslant 20$ possess a maximum at $\lambda_{c}^{*} \approx 10$.

To study the scaling behavior in the weakly attractive regime, we introduce the effective interaction parameter $\lambda^{*}$ $=\lambda N^{\phi}$. Figure 3 depicts $R_{g}^{2}$ as a function of $\lambda^{*}$. As is obvious from the figure, the data for various chain lengths collapse approximately onto a universal curve, at least for $\lambda^{*}$ $<100$. (By changing the exponent $\nu$ by less than $2 \%$ for some of the curves, an even better agreement can be achieved.) Hence, the two chains only partially intervene each other for $\lambda^{*}<100$. There is a certain overlap of the two chains but the mean separation of the monomers is still of the order of the radius of gyration. Clearly, there is a tendency of the systems to start collapsing approximately at the same value of $\lambda^{*}=\lambda_{c}^{*} \approx 10$. This coincidence underlines our assumption that $\lambda^{*}$ is the appropriate parameter to compare chains of various lengths. The chain characteristics $R_{g}$ and $R$ obey approximately the scaling relations $R_{g}^{2} \sim(N-1)^{2 \nu}$ (cf. Fig. 3) and $R^{2} \sim(N-1)^{2 \nu}$, respectively. But despite the weak stretching of the chains, we find practically the same exponent $\nu$ for all interaction strengths.

Single polyelectrolyte chains in the presence of counterions exhibit a different behavior [8]. Such chains also start to collapse at a certain interaction strength $(\lambda)$ due to counterion condensation. However, the corresponding $\lambda$ value is nearly independent of chain length. Considering the exponents $(\nu)$ for the mean square end-to-end distance and the radius of gyration, respectively, for these systems, the exponents increase with increasing interaction strength until counterion condensation starts and the exponent for the mean square end-to-end distance is close to $\nu \approx 1$ corresponding to rodlike chains. The chains in the two-chain system, however, are only weakly perturbed for $\lambda^{*}<100$, because of the effectively small interaction strength $\lambda$. In particular, they are never stretched in a rodlike manner due to the screening of the intramolecular Coulomb interaction by the oppositely charged chains. For larger interaction parameters, the chains start to collapse and start to form dense clusters.

The critical effective interaction strength $\lambda_{c}^{*}$ can be used to separate the one-phase state with scattered chains and the phase with a globule. For $\lambda^{*}<\lambda_{c}^{*}$ individual chains are 

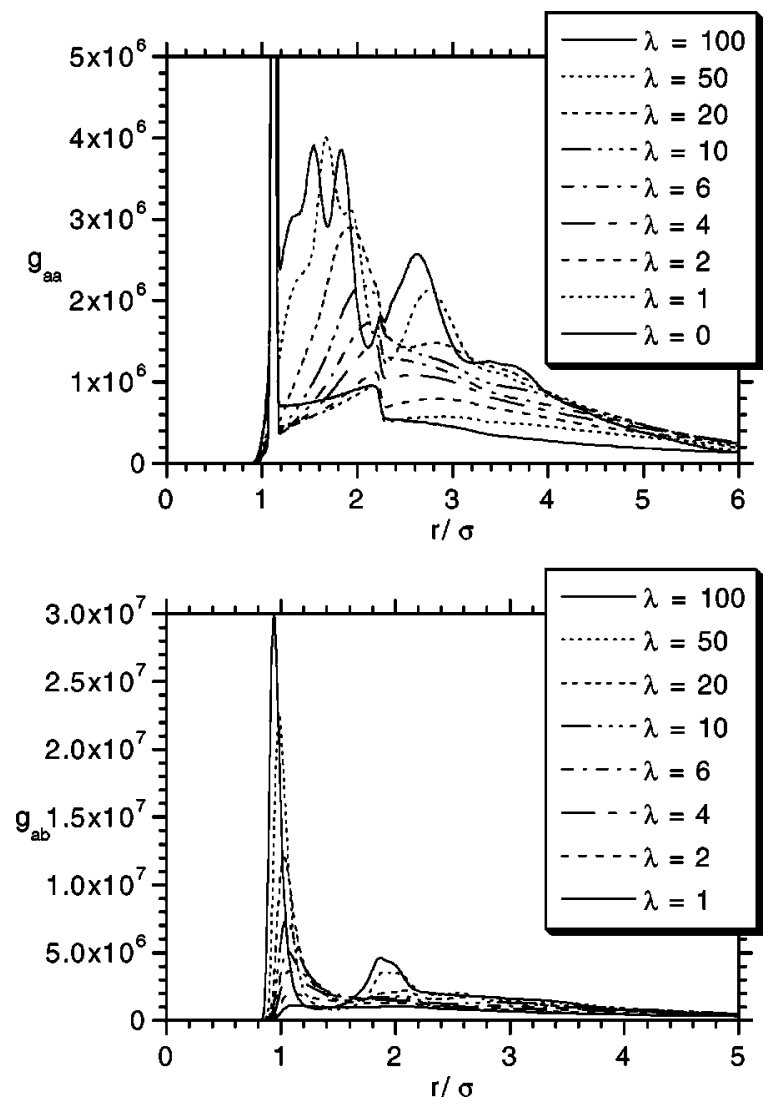

FIG. 4. Pair correlation functions for chains of length $N=80$ and various interaction strengths. Top: intramolecular pair correlation function; bottom: intermolecular pair correlation function.

present, in contrast, for $\lambda^{*}>\lambda_{c}^{*}$ the chains aggregate and fold into densely packed structures.

\section{Large values of $\lambda$}

With increasing interaction parameter the chains eventually form very compact, collapsed objects. In this range of strong interactions, $\lambda^{*} \gg \lambda_{c}^{*}$, the chain systems again show a scaling behavior for certain characteristic length scales. For values of $\lambda$ larger than 1 we find for the radius of gyration $\nu \approx 1 / 3$, corresponding to the obvious compact structure.

In the aggregated state, we compared chains of different lengths at the same value of $\lambda$ and not at the same value $\lambda *$. This is reasonable, since in the condensed state screening is present. The monomers of the two chains form pairs leading to an effective dipolar interaction among the different pairs [8]. The observed scaling behavior of the radius of gyration confirms this approach.

Our results are in agreement with the results obtained in Ref. [12]. Depending on the interaction strength the authors of Ref. [12] find $\nu$ values in the range $0.35-0.5$.

Randomly charged neutral polyampholytes also exhibit a collapse and dense structures are formed for sufficiently large interaction strengths or, vice versa, sufficiently small temperatures. However, the molecular weight dependence of the critical temperature (or interaction strength $\lambda_{c}^{*}$ ) separating the regimes of individual chains from clusters of chains
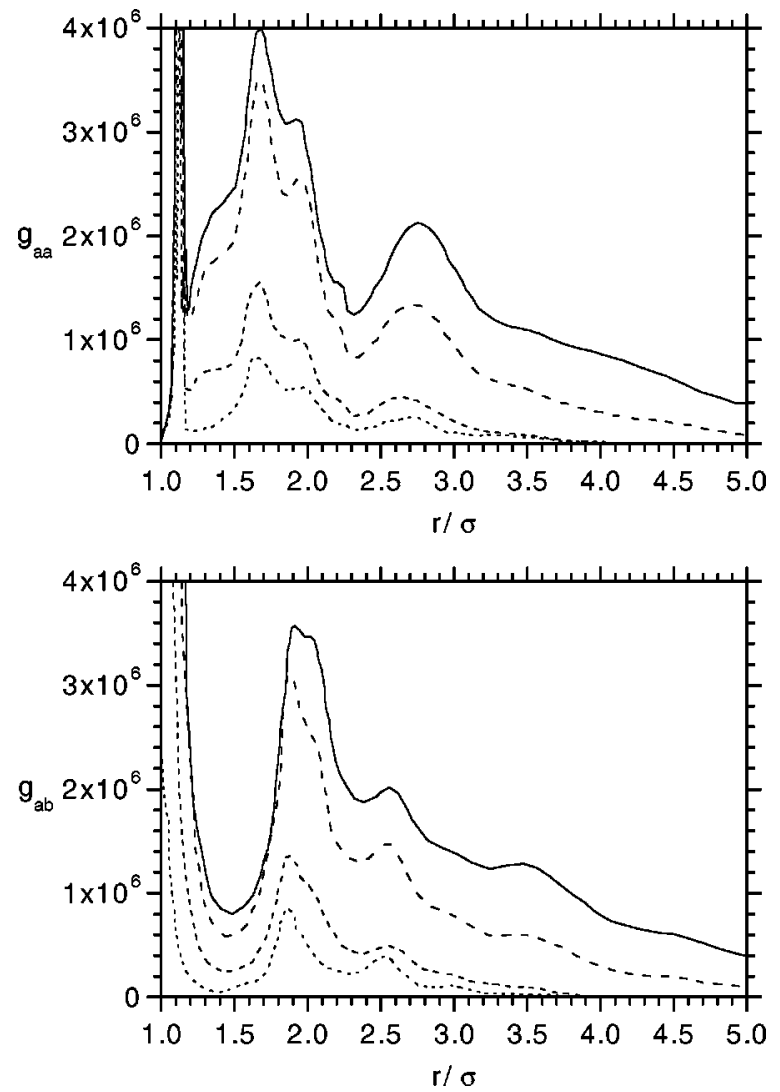

FIG. 5. Pair correlation functions for the interaction strength $\lambda$ $=50$ and the chain lengths $N=10,20,40,80$ (from bottom to top). Top: intramolecular pair correlation function; bottom: intermolecular pair correlation function.

is quite different. Using a similar criterion for random polyampholyte chains that are globally neutral as for the polyelectrolyte system, the Coulomb energy is given by $E_{C}$ $\sim e^{2} N / R_{g} \sim e^{2} N / N^{0.5-0.6} \quad[22,26]$. The critical interaction strength is then proportional to $N^{0.4-0.5}$. As demonstrated by our simulations, the critical interaction strength for the aggregation of oppositely charged polyelectrolytes is proportional to $N^{1.4}$. Moreover, the chain length dependence of the

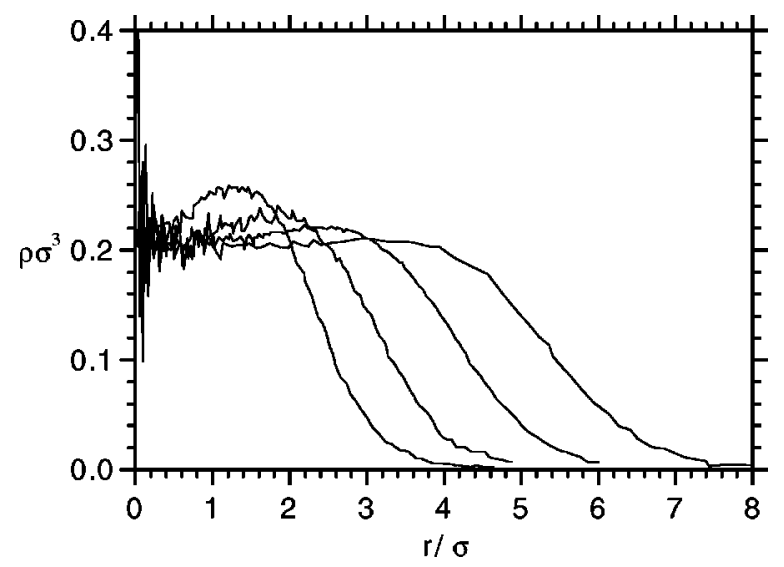

FIG. 6. Radial density of monomers with respect to the center of mass of a cluster. The interaction strength is $\lambda=4$ and the chain lengths are $N=20,40,80,160$ (from left to right). 

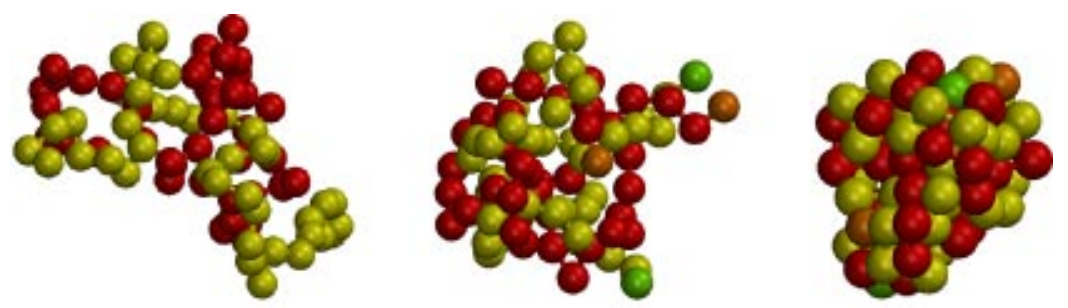

FIG. 7. Clusters for chains of length $N=40$ and the interaction strengths $\lambda=0.4,4,40$ (from left to right).

probability for the polyelectrolyte globule to be destroyed by random thermal agitation is approximately given by $P$ $\sim \exp \left(-N^{\phi}\right)(\phi=1.4)$, as a consequence of the effective interaction parameter $\lambda^{*}$. Hence, the aggregation of the chains in a polyelectrolyte globule is stronger than in a random polyampholyte globule, where $P \sim \exp \left(-N^{0.4-0.5}\right)$. Thus, polyelectrolyte globules are formed at lower critical temperatures and they will be even more stable than random polyampholyte globules.

\section{B. Pair correlation function}

The local structure of the aggregated chains is analyzed by the pair correlation function. As shown by Fig. 4, the intramolecular pair correlation function $\left(g_{a a}\right)$ and the intermolecular pair correlation function $\left(g_{a b}\right)$, respectively, exhibit distinct peaks indicating a strong aggregation of the oppositely charged monomers with increasing interaction strength. Basically the same local structures are observed for strong interactions, independent of the size of the systems, as is obvious from Fig. 5. The whole system gradually freezes with increasing interaction strength and the molecules form a glasslike structure. Pronounced peaks at very large interaction strengths indicate the formation of local order. Locally (on the length scale of only a very few monomers) densely packed structures are formed, which are similar to structures present in crystals. A phase transition into a crystalline state of the whole system is prevented by the bonds of the chains. The cluster is a frustrated system due to the presence of the bonds. A similar behavior is observed in randomly charged polyampholytes $[16,17,21,22]$.

Depending on the extensibility of the bonds, crystalline or glasslike structures have been reported for systems of randomly charged neutral polyampholytes [24]. In particular, the glasslike state is obtained for finite extensible bonds. This is in agreement with our simulations, because the bonds of our model chains are essentially inextensible.

\section{Monomer density within clusters}

In Ref. [35] a statistical theory of globular polyelectrolyte complexes is presented. In particular, the density of monomers within the globule is estimated assuming that the density is constant. Although our simulated systems are not identical to the system investigated in Ref. [35], we analyzed the monomer density profiles for weak interactions.

Our simulations exhibit plateaus in the monomer density profiles for interaction strengths $\lambda<20$, confirming the assumption of the above authors. The radial extension of the plateau depends naturally on the chain length. For $\lambda>20$, densely packed structures are formed and the radial density profile exhibits a peak structure corresponding to preferred positions of individual monomers. The average density reaches an interaction independent limiting value for $\lambda>50$, which is larger than the density at $\lambda=20$.

In the regime $0.6<\lambda<20$, a comparison shows that the density is independent of the chain length within the accuracy of our simulations. Figure 6 presents an example for $\lambda$
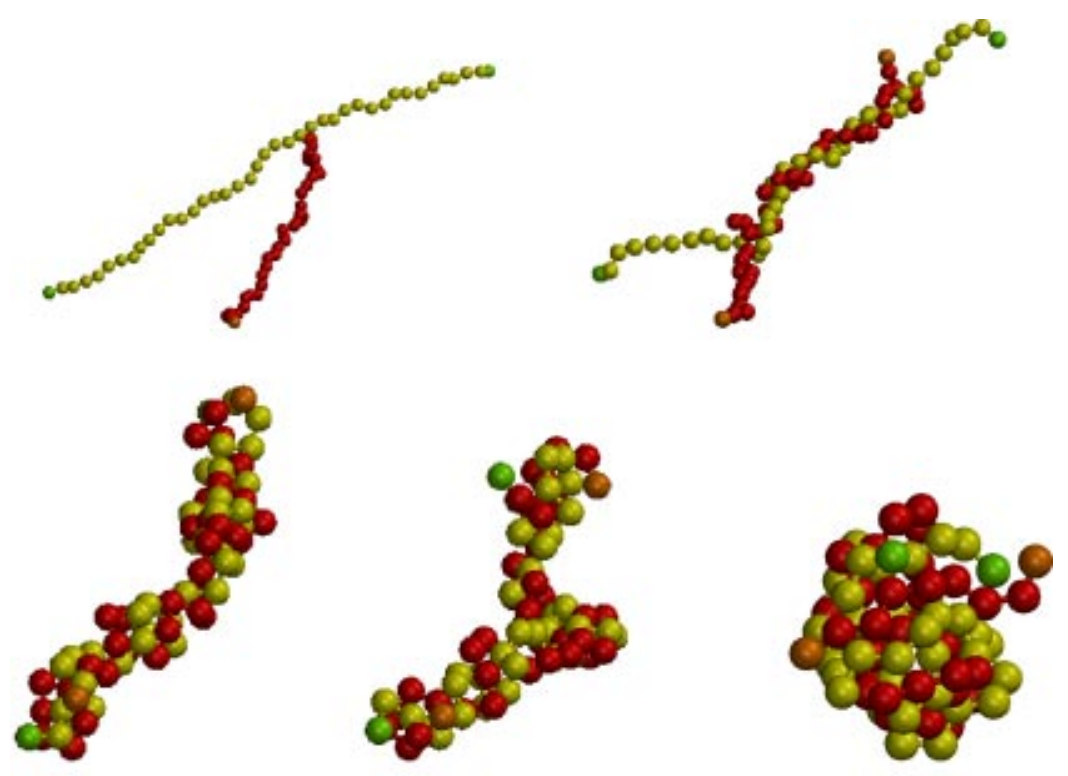

FIG. 8. Successive conformations of chains of length $N=40$ at the interaction strength $\lambda=8$ during the formation of a globule; $t / \tau=0,6$ $\times 10^{4}, \quad 1.2 \times 10^{5}, \quad 4.75 \times 10^{5}$, and equilibrium state (from top left to bottom right). 


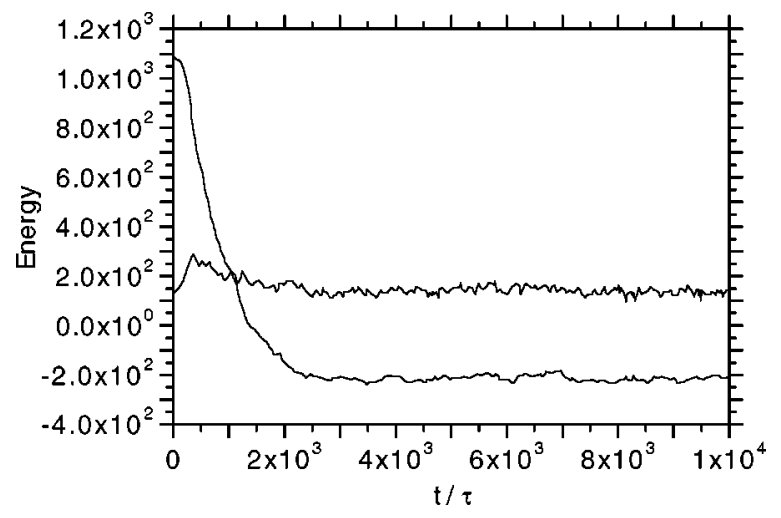

FIG. 9. Time dependence of the kinetic (solid line) and potential (dashed line) energy during the formation of a globule $(N$ $=40 ; \lambda=8)$.

$=4.0$. The plateaulike regime is clearly present for long chains. For short chains we observe a pronounced maximum of the density. This maximum vanishes with decreasing interaction strength. On the other hand, such a maximum appears also at higher interaction strengths for the longer chains. The reason for this is the geometry of the globule. Monomers on the outer part of the cluster experience an attractive interaction from inside the cluster and no interaction from outside. Thus, these monomers show a stronger attraction by the kernel part than monomers inside of the cluster. For smaller interaction strengths (or higher temperatures) chains of different lengths are deformed to different degrees (cf. Fig. 1), which leads to a chain length dependence of the density profile. Within the regime $0.6<\lambda<20$, the density $\rho$ exhibits the scaling relation $\rho \sim \lambda^{0.37}$ very accurately. The exponent is quite different from the predictions made in Ref. [35]. For a salt-free solution, Eq. (25) of Ref. [35] yields a monotonously increasing density as a function of the interaction strength. In the limit $\lambda \rightarrow 0$ the relation $\rho$ $\sim \lambda^{3 / 5}$ is obtained and in the opposite limit $\lambda \rightarrow \infty$ the relation $\rho \sim \lambda^{3}$ follows. Hence, the exponents are always larger than that found in our simulations.

Figure 7 illustrates the density of the clusters for different interaction strengths $\lambda(=0.4,4,40)$. For the chain length $N=40$ no compact structure is formed at $\lambda=0.4$, although the radius of gyration of a chain is already smaller than in a good solvent (cf. Fig. 1). At $\lambda=4.0$ there is already a pronounced peak in the pair correlation function $g_{a a}$ at $r=2 \sigma$ (cf. Fig 4) indicating the formation of a denser structure. A further increase of $\lambda$ leads to an even more compact cluster. The local crystallinelike structure, however, is obtained for even larger interaction strengths $(\lambda>50)$.

\section{KINETICS OF CLUSTER FORMATION}

We start our simulations with well separated chains. Turning on the Coulomb interaction, the chains attract each other. In a first step of the aggregation process-at sufficiently large interaction strengths - the chains start to wind around each other and form helical-like superstructures similar to the snapshots presented in Figs. 2 and 8, respectively. However,

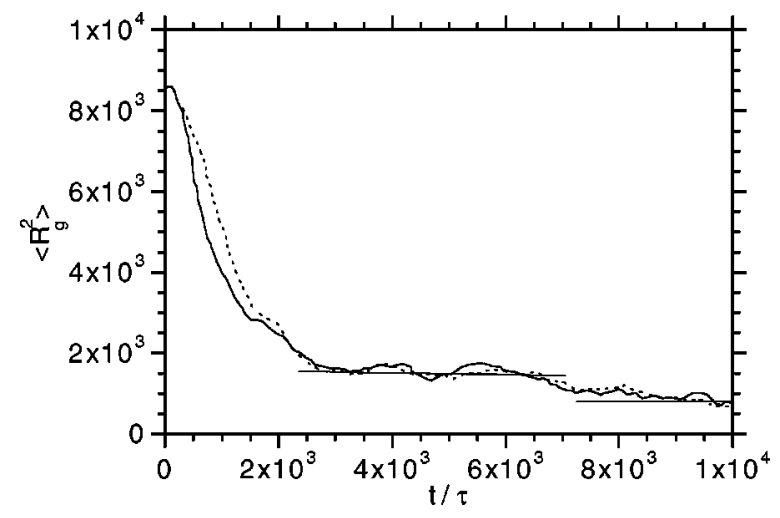

FIG. 10. Time dependence of the radius of gyration of two chains during the formation of a globule $(N=40 ; \lambda=8)$. The decrease of $R_{g}^{2}$ at $t / \tau \approx 6.5 \times 10^{3}$ indicates a reshuffling of the monomers within the cluster.

by inspection, we see that the chains do not form perfect helices. In particular, there is no defined helicity along the whole molecular string (except for chains with $N=10$ ). Instead, the winding direction changes along the string. This is supported by the flexibility of the chain. In a second step the chains fold into a compact structure because of the attractive interaction between dipoles formed by oppositely charged monomers (cf. Fig. 8). The imperfect helix supports the formation of the compact aggregate. Due to the lack of the perfect twist, monomers and groups of monomers are rather mobile and can therefore easily rearrange to form the cluster. To untwist a perfect helix before the collapse would require more time than for a disordered helix. Indeed, the folding process of the system is very fast, as is obvious from Fig. 9.

Figure 9 displays the time dependence of the kinetic and potential energy during the folding process. The kinetic energy is essentially constant since the system is coupled to a heat bath by stochastic forces. The potential energy rapidly decreases and assumes for $t>2.5 \times 10^{3} \tau$ a constant value.

Figure 10 exhibits the changes of the radius of gyration of the two chains. $R_{g}^{2}$ decreases on the same time scale as the potential energy. Between $t=2.5 \times 10^{3} \tau$ and $t=6.5 \times 10^{3} \tau$ the radius of gyration is approximately constant. At $t \approx 6.5$ $\times 10^{3} \tau$ conformational changes occur in the system leading to smaller values of $R_{g}^{2}$ for both chains. The potential energy is obviously unaffected by these changes, which leads us to conclude that the conformational changes are only of entropic nature.

The whole aggregation process-starting from the approach of the two chains, the helix formation, and the folding-is a cooperative process. This is obvious from the snapshots of Fig. 8 as well as the time dependence of the radius of gyration.

\section{CONCLUSIONS}

We have presented molecular dynamics simulations of systems with two oppositely charged chains. For small interaction strengths, we find that similar structures for chains of various lengths are obtained for the effective interaction strength $\lambda *=\lambda N^{1.4}$. In this regime the chains exhibit univer- 
sal behavior and we find scaling exponents for the radius of gyration and the end-to-end distance similar to the values for an uncharged chain in a good solvent. As soon as the interaction is strong enough, the systems collapse and form very compact glasslike (for large enough $\lambda$ ) structures with a distinct round surface and pronounced local order. This occurs at all investigated chain lengths. Systems of various chain lengths exhibit again self-similarity on small length scale. Collective phenomena play a major role in the formation of the observed structures, starting from the moment the chains begin to wind around each other, subsequently form helicallike structures, and finally upto the moment when they fold into dense globules.

In addition, we performed preliminary simulations taking into account the counterions. These simulations suggest that the counterions possess no significant influence on the formation of the aggregate. The charge density of the chains is very large leading to the formation of aggregates even at very small $\lambda$. For example, chains of length $N=40$ start aggregating at $\lambda \approx 0.05$. This interaction strength is by far too small to lead to a condensation of counterions on a chain [8]. At higher interaction strengths more compact cluster and counterion pairs are formed.

We presented results for two oppositely charged chains. We expected the observed behavior to apply for dilute systems, where the density is much smaller than the overlap density. This is confirmed by Monte Carlo simulations of Ref. [32].

In future studies we will extend this simulations to systems with more than two chains and denser systems. In such systems several chains of the same charge will aggregate with an oppositely charged chain. The competition among the various chains will lead to interesting self-organized structures [32].
[1] J.-L. Barrat and J.-F. Joanny, Adv. Chem. Phys. XCIV, 1 (1996).

[2] M. Hara, Polyelectrolytes: Science and Technology (Marcel Dekker, New York, 1993)

[3] Macro-ion Characterization: From Dilute Solutions to Complex Fluids, edited by K. Schmitz (American Chemical Society, Washington, DC, 1994).

[4] S. Förster and M. Schmid, Adv. Polym. Sci. 120, 51 (1995).

[5] M. Stevens and K. Kremer, J. Chem. Phys. 103, 1669 (1995).

[6] M. Stevens and K. Kremer, J. Phys. II 6, 1607 (1996).

[7] D. Stigter, Macromolecules 95, 380 (1995).

[8] R. G. Winkler, M. Gold, and P. Reineker, Phys. Rev. Lett. 80, 3731 (1995).

[9] A. V. Dobrynin and M. Rubinstein, Macromolecules 29, 2974 (1996).

[10] Y. Yamasaki, Y. Teramoto, and K. Yoshikawa, Biophys. J. 80, 2823 (2001).

[11] P. L. Felgner et al., Proc. Natl. Acad. Sci. U.S.A. 84, 7413 (1987).

[12] D. Srivastava and M. Muthukumar, Macromolecules 27, 1461 (1994).

[13] S. F. Edwards, P. R. King, and P. Pincus, Ferroelectrics 30, 3 (1980)

[14] P. Higgs and J.-F. Joanny, J. Chem. Phys. 94, 1543 (1991).

[15] Y. Kantor, H. Li, and M. Kardar, Phys. Rev. Lett. 69, 61 (1992).

[16] Y. Kantor, M. Kardar, and H. Li, Phys. Rev. E 49, 1383 (1994).

[17] M. Schulz, R. G. Winkler, and P. Reineker, Phys. Rev. Lett. 73, 1602 (1994).
[18] A. M. Gutin and E. Shakhnovich, Phys. Rev. E 50, R3322 (1994).

[19] A. V. Dobrynin and M. Rubinstein, J. Phys. II 5, 677 (1995).

[20] R. Everaers, A. Johner, and J.-F. Joanny, Europhys. Lett. 37, 275 (1997).

[21] M. Tanaka, A. Yu. Grosberg, V. S. Pande, and T. Tanaka, Phys. Rev. E 56, 5798 (1997).

[22] M. Tanaka, A. Yu. Grosberg, and T. Tanaka, J. Chem. Phys. 110, 8176 (1999).

[23] J. B. Imbert, J. M. Victor, N. Tsunekawa, and Y. Hiwatari, Phys. Lett. A 258, 92 (1999).

[24] M. Tanaka and T. Tanaka, Phys. Rev. E 62, 3803 (2000).

[25] Y. Kantor and M. Kardar, Phys. Rev. E 52, 835 (1995).

[26] V. Yamakov, A. Milchev, H. J. Limbach, B. Dünweg, and R. Everaers, Phys. Rev. Lett. 85, 4305 (2000).

[27] R. G. Winkler and P. Reineker, J. Chem. Phys. 106, 2841 (1997).

[28] H. Schiessel and A. Blumen, J. Chem. Phys. 104, 6036 (1996).

[29] T. Soddemann, H. Schiessel, and A. Blumen, Phys. Rev. E 57, 2081 (1998).

[30] G. Whitesides and C. Seto, Science 254, 1312 (1991).

[31] R. Lakes, Science 361, 511 (1993).

[32] Y. Hayashi, M. Ullner, and P. Linse, J. Chem. Phys. 116, 6836 (2002).

[33] R. G. Winkler, P. J. Ludovice, D. Y. Yoon, and H. Morawitz, J. Chem. Phys. 95, 4709 (1991).

[34] H. Risken, The Fokker-Planck Equation (Springer-Verlag, Berlin, 1989).

[35] V. Yu. Borue and I. Ya. Erukhimovich, Macromolecules 23, 3625 (1990). 